\title{
Satisfacción del paciente postquirúrgico ante los cuidados de Enfermería
}

\author{
Post-surgical Patient Satisfaction with Nursing Care
}

\author{
Chávez Ramírez Karina ${ }^{1}$; López Escudero Alma Belém ${ }^{2}$; Hernández Montiel Miguel Ángel ${ }^{3}$; \\ Sánchez Tlatilolpa Estephany Alejandra ${ }^{4}$; Sanchez Padilla $M^{5}{ }^{5}$; Arias R. ${ }^{6}$; Lazcano Ortiz \\ Margarita $^{7}$
}

\begin{abstract}
:
Objective: To determine the level of satisfaction that post-surgical patients refer to the care provided by the professional nursing.

Material and method: Design of the non-experimental study, quantitative kind, descriptive transversal. Non-probabilistic sampling to convenience, the sample was 15 postoperative patients operated at the General Hospital de Pachuca, Hgo., The test "Satisfaction of the postoperative patient before nursing care" was applied with 28 items. To measure the level of satisfaction, the "Patient Satisfaction Scale with Nursing Care" was used, with Cronbach's Alpha of 0.92.

Results: The average age is 48 years SD (5.3); 8 (53.3\%) are men; 4 (26.7\%) from Pachuca, $40 \%$ have more than four days of stay in the hospital. $86 \%$ showed a high level of satisfaction.

Conclusions: It is concluded that the level of satisfaction of patients with the nursing care of the General Hospital de Pachuca, Hgo., In the postoperative stage is high $(86.6 \%)$.

Keywords:

Patient satisfaction, nursing care, post-surgical patient, mature adult

\section{Resumen:}

Objetivo: Determinar el nivel de satisfacción que los pacientes post-quirúrgicos refieren ante los cuidados brindados por el profesional de enfermería. Material y método: Diseño del estudio no experimental, tipo cuantitativo, descriptivo transversal. Muestreo no probabilístico por conveniencia, la muestra fue de 15 pacientes posquirúrgicos intervenidos en el Hospital General de Pachuca, Hgo., se aplicó el cuestionario "Satisfacción del paciente posquirúrgico ante los cuidados de enfermería" con 28 ítems. Para medir el nivel de satisfacción se utilizó la "Escala de Satisfacción del paciente con el cuidado de enfermería", con Alfa de Cronbach de 0.92. Resultados: El promedio de edad es de 48 años, DE (5.3); 8 (53.3\%) son hombres; 4 (26.7\%) provenientes de Pachuca, el $40 \%$ tiene
\end{abstract}

\footnotetext{
${ }^{1}$ Alumnos del Área Académica de Enfermería del Instituto de Ciencias de la Salud de la Universidad Autónoma del Estado de Hidalgo.

${ }^{2}$ Alumnos del Área Académica de Enfermería del Instituto de Ciencias de la Salud de la Universidad Autónoma del Estado de Hidalgo.

${ }^{3}$ Alumnos del Área Académica de Enfermería del Instituto de Ciencias de la Salud de la Universidad Autónoma del Estado de Hidalgo.

${ }^{4}$ Alumnos del Área Académica de Enfermería del Instituto de Ciencias de la Salud de la Universidad Autónoma del Estado de Hidalgo.

${ }^{5}$ Profesor investigador de tiempo completo; Área Académica de Enfermería del Instituto de Ciencias de la Salud de la Universidad Autónoma del Estado de Hidalgo.

${ }^{6}$ Profesor investigador de tiempo completo; Área Académica de Enfermería del Instituto de Ciencias de la Salud de la Universidad Autónoma del Estado de Hidalgo.

${ }^{7}$ Profesor investigador de tiempo completo; Área Académica de Enfermería del Instituto de Ciencias de la Salud de la Universidad Autónoma del Estado de Hidalgo. Email: magos1000@ hotmail.com
} 
más de cuatro días de estancia dentro del hospital. El 86\% obtuvo un nivel de satisfacción Alto. Conclusiones: El nivel de satisfacción de los pacientes con la atención de enfermería del Hospital General de Pachuca, Hgo., en la etapa posoperatoria es alto (86.6\%).

\section{Palabras Clave:}

Satisfacción del paciente, cuidado de enfermería, paciente posquirúrgico, adulto maduro

\section{Marco Teórico}

La atención con calidad y calidez de la enfermera en el postoperatorio; es básica y fundamental, ya que de ello depende el éxito o fracaso de la recuperación del paciente. El someterse a una intervención quirúrgica es algo que al paciente le causa miedo, ansiedad, y estrés; por lo que el profesional de enfermería debe brindar atención individualizada a cada paciente con la finalidad de prepararlo para el acto quirúrgico y que éste se presente en las mejores condiciones posibles, tanto físicas como psicológicas ${ }^{1}$

En el Hospital General de Pachuca se estima que cada año se practican 15 mil cirugías ${ }^{2}$

Por lo que el evaluar los cuidados de enfermería y el nivel de satisfacción de los pacientes permitirá la identificación de potencialidades y deficiencias en el campo del estudio, para la reorganización de las actividades asistenciales, gerenciales y de enseñanza ${ }^{3}$

Los pacientes post-quirúrgicos, en su mayoría, presentan signos y síntomas, tales como, la presencia de dolor desde leve a intenso, deterioro de la movilidad, existencia de herida quirúrgica, disminución de la función intestinal, déficit en el autocuidado como el aspecto de higiene, alimentación, baño, entre muchas otras afecciones. Por lo tanto, la atención brindada por el personal de enfermería debe ser completa y acorde a las necesidades del paciente, tomando en cuenta que para poder realizar esto es necesario tener conocimientos teóricos, científicos y prácticos, siendo evaluada la atención con base a la satisfacción del paciente.

La satisfacción es un estado de la mente, generado por una optimización de la retroalimentación cerebral, por la cual las diferentes zonas cerebrales compensan el potencial energético que lleve a cabo el cerebro, es decir, cuanto mayor sea la capacidad de neurotransmisor mayor será la posibilidad de satisfacción.

En el paciente es una sensación subjetiva que experimenta este, al colmar sus necesidades y expectativas cuando se le otorga un servicio. Está subordinada a numerosos factores, entre los que se incluyen las experiencias previas, las expectativas y la información recibida de otros usuarios y del propio establecimiento de salud. La satisfacción del paciente en el cuidado de enfermería, fortalece la confianza en los cuidados que el profesional le brinda en el proceso saludenfermedad para su pronta recuperación y la reincorporación en el núcleo familiar.

El cuidado es la esencia de la profesión de enfermería, el cual se puede definir como: una actividad que requiere de un valor personal y profesional encaminado a la conservación, restablecimiento y auto cuidado de la vida que se fundamenta en la relación terapéutica enfermera- paciente. Es sabido que el cuidar, es una actividad indispensable para la supervivencia, desde que la humanidad existe, el cuidado ha sido relevante constituyendo una función primordial para promover y desarrollar todas aquellas actividades que hacen vivir a las personas y a los grupos.

Del Modelo de Calidad de la Atención Médica de Avedis Donabedian se destaca lo más importante de la introducción de conceptos como: Estructura, Proceso y Resultado, que constituyen hoy en día el paradigma dominante de evaluación de la calidad de la atención a la salud. La idea que los pacientes deben involucrarse en su atención en general no es practicada de manera responsable. Hoy día la gente habla de autonomía del paciente, pero por lo general eso se traduce en abandono del paciente. Se retomaran los indicadores de resultado que se encuentran referidos a la variación de los niveles de salud y a la satisfacción del paciente por la atención recibida, la cual le otorga una importancia mayor por la necesidad de satisfacer las expectativas del usuario mediante un servicio de mayor calidad ${ }^{4}$

\section{Objetivo}

Determinar el nivel de satisfacción que los pacientes postquirúrgicos refieren ante los cuidados brindados por el profesional de enfermería.

\section{Material y método}

El diseño de la investigación es no experimental, de tipo cuantitativo, descriptivo transversal. Muestreo no probabilístico de conveniencia, la muestra fue de 15 pacientes en etapa posquirúrgica, hospitalizados en el servicio de Cirugía del Hospital General de Pachuca, Hgo.

Los datos fueron recolectados mediante la aplicación del cuestionario "Satisfacción del paciente posquirúrgico ante los cuidados de enfermería" compuesto por preguntas socio demográficas, generales sobre la cirugía y estancia hospitalaria, con relación al cuidado que enfermería brinda al paciente y sobre la satisfacción del mismo. Simultáneamente se aplicó la "Escala de Satisfacción del Paciente con el Cuidado de Enfermería" que está conformada por 29 preguntas que abarcan cinco dimensiones: trato, calidad técnica del cuidado, disponibilidad, continuidad y resultado del cuidado. Con opciones de respuesta siendo Nunca 1 hasta Siempre 4. Obteniendo un Alfa de Crombach de 0.92, considerándose un instrumento confiable. El nivel de satisfacción es definido de acuerdo a la puntuación: alto (88-116), mediano $(59-87)$ y bajo $(29,58)$. Se utilizó para el análisis la base de datos electrónica Statistical 
Package for the Social Sciences (SPSS 22.0); estadística descriptiva y elaboración de gráficas y tablas.

\section{Resultados}

Se verificó en el análisis del perfil sociodemográfico de los entrevistados, que la media de edad fue de 48 años, DE (5.3) y la moda de 43 años. De los 15 entrevistados, $8(53.3 \%)$ hombres y 7 (46.7\%) mujeres; 4 (26.7\%) eran originarios de Pachuca, Hgo, el resto provenientes de municipios aledaños y 1 (6.7\%) de otro estado; 7 (46.7\%) con nivel básico de escolaridad y 1 (6.7\%) profesional. Cabe mencionar que $4(26.7 \%)$ han tenido más de cuatro intervenciones quirúrgicas, y $6(40 \%)$ tienen más de cuatro días de estancia hospitalaria, representando los datos en la siguiente tabla:

\begin{tabular}{lccccc} 
Tabla 1. Número de intervenciones quirúrgicas y dias de estancia hospitalaria. \\
\hline $\begin{array}{l}\text { Número de intervenciones } \\
\text { quirúrgicas }\end{array}$ & Frecuencia & Porcentaje & $\begin{array}{c}\text { Días de estancia } \\
\text { hospitalaria }\end{array}$ & Frecuencia & Porcentaje \\
\hline $\begin{array}{l}\text { Una intervención } \\
\text { Dos Intervenciones }\end{array}$ & 7 & 46.7 & Un día & 2 & 13.3 \\
Tres Intervenciones & 2 & 13.3 & Dos días & 4 & 26.7 \\
Cuatro Intervenciones o & 2 & 13.3 & Tres dias & 3 & 20.0 \\
mas & 4 & 28.7 & Cuatro dias o mas & 6 & 40.0 \\
Total & 15 & 100.0 & Total & 15 & 100.0 \\
\hline Fuente: Cuestionario "Satisfacción del paciente postquirúrgico ante los cuidados de enfermeria".
\end{tabular}

En cuanto a los datos de interés y con mayor relevancia analizados y representados en las tablas 2 y 3 se obtiene que la mayoría de pacientes (93.3\%), afirman que las enfermeras han realizado la curación de su herida quirúrgica de manera cuidadosa y les han resuelto dudas sobre su estado de salud.

Tabla 2.- Las enfermeras le realizan la curación de su herida quirúrgica de manera:

\begin{tabular}{|c|c|c|}
\hline & Frecuencia & Porcentaje \\
\hline Cuidadosa & 14 & 93.3 \\
\hline Brusca & 1 & 6.7 \\
\hline Total & 15 & 100.0 \\
\hline \multicolumn{3}{|c|}{$\begin{array}{l}\text { Fuente: Cuestionario "Satisfacción del } \\
\text { paciente postquirúrgico ante los cuidados de } \\
\text { enfermeria". }\end{array}$} \\
\hline \multicolumn{3}{|c|}{$\begin{array}{l}\text { Tabla 3. ¿Las enfermeras le resuelven dudas } \\
\text { sobre su estado de salud? }\end{array}$} \\
\hline & Frecuencia & Porcentaje \\
\hline $\mathbf{S i}$ & 14 & 93.3 \\
\hline No & 1 & 6.7 \\
\hline Total & 15 & 100.0 \\
\hline \multicolumn{3}{|c|}{$\begin{array}{l}\text { Fuente: Cuestionario "Satisfacción del } \\
\text { paciente postquirúrgico ante los cuidados de } \\
\text { enfermeria". }\end{array}$} \\
\hline
\end{tabular}

Mientras que el $73.3 \%$ de pacientes afirman que el personal de enfermería les ayuda a movilizarse cuando lo requieren y también que mantienen su cama limpia
Tabla 4.- ¿Las enfermeras le ayudan a movilizarse cuando usted lo requiere?

\begin{tabular}{lcc}
\hline & Frecuencia & Porcentaje \\
\hline Si & 11 & 73.3 \\
No & 4 & 26.7 \\
Total & 15 & 100.0 \\
\hline
\end{tabular}

Fuente: Cuestionario "Satisfacción del paciente postquirúrgico ante los cuidados de enfermería".

Tabla 5.- Las enfermeras mantienen su cama:

\begin{tabular}{lcc}
\hline & Frecuencia & Porcentaje \\
\hline Limpia y ordenada & 11 & 73.3 \\
Solo limpia & 4 & 26.7 \\
Total & 15 & 100.0 \\
\hline Fuente: Cuestionario & "Satisfacción del & paciente \\
postquirúrgico ante los cuidados de enfermería".
\end{tabular}

De acuerdo a los resultados de la tabla 6 , se muestra que $13(86.6 \%)$ pacientes tienen un nivel alto de satisfacción, mientras que $1(6.7 \%)$ tiene un nivel mediano de satisfacción y el resto un nivel bajo.

Tabla 6. Nivel de satisfacción.

\begin{tabular}{lcc}
\hline & Frecuencia & Porcentaje \\
\hline Alto & 13 & 86.6 \\
Mediano & 1 & 6.7 \\
Bajo & 1 & 6.7 \\
& & 100 \\
\hline
\end{tabular}

Con lo expuesto anteriormente obtenido del instrumento "Escala de satisfacción del paciente con el cuidado de enfermería", se analizó y representó estadísticamente que; de los 15 participantes, 13 (86.6\%) se encuentran con un Alto nivel de Satisfacción ante los cuidados que el profesional de enfermería les proporciona durante su estancia hospitalaria, mientras que 2 (13.4\%) se encuentran con un nivel Mediano y Bajo de satisfacción. 


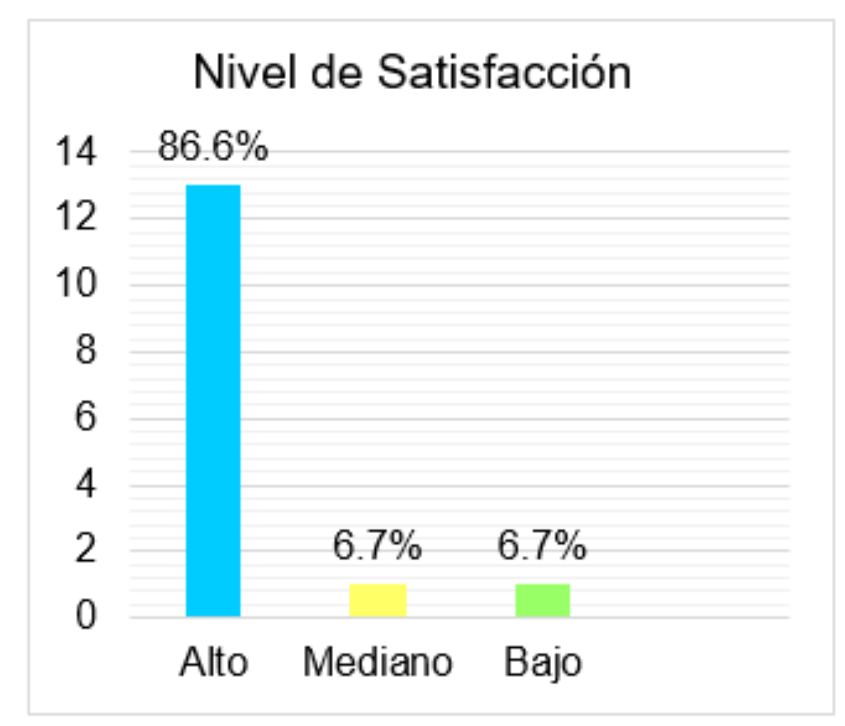

Discusión

De acuerdo a los resultados obtenidos en este estudio, se encontró similitud con un estudio ${ }^{5}$ que presenta como resultado de su investigación un nivel alto de satisfacción en los pacientes posquirúrgicos con el cuidado de enfermería. A diferencia con estudios realizados ${ }^{2}$ donde los resultados de su investigación demostraron un nivel parcial de satisfacción del paciente ante los cuidados de enfermería; Otro estudio presento que la satisfacción del paciente posoperado frente a los cuidados del profesional de enfermería es de un nivel medio y otro obtuvo resultados de satisfacción en un nivel bajo ${ }^{6}$ con base en los cuidados de enfermería.

Siendo un punto clave la satisfacción que tienen los pacientes, ya que la misión de las instituciones hospitalarias es brindar atención de calidad y calidez a los usuarios, independientemente de su género, edad o nivel académico. La información referida por los pacientes acerca de su percepción de la calidad de la atención que se les brinda, es una herramienta clave para la implementación de estrategias de mejora continua.

\section{Conclusiones}

Los resultados encontrados en este estudio indican que un mayor porcentaje de los pacientes entrevistados presentan un nivel alto de satisfacción ante el cuidado que los profesionales de enfermería les brindan en su estancia postoperatoria, debido a que la atención prestada a los pacientes posquirúrgicos es adecuada al presentarse el profesional de enfermería con el paciente y tratarlo de forma respetuosa, así como aclarando dudas sobre alguna cuestión que el paciente externe de manera clara, se sienten satisfechos porque las enfermeras son amables y eficientes, ya que siempre hay una enfermera disponible para cuando el paciente la requiere y realizan sus procedimientos con mucha habilidad y organización manteniendo su entorno limpio y ordenado.

\section{Referencia}

1.Torres, Y. Hospital general, en dos meses, estima director. (Internet) 2007 (citado 2018 mayo 12) Recuperado de: http://www.criteriohidalgo.com/noticias/hidalgo-ujul/hospital-generalen-dos-meses-estima-director

2. Velazco, U. \& Natividad, I. Satisfacción de los pacientes con la atención de enfermería postoperatoria en el Servicio de Cirugía del Hospital "Santa María del Socorro".(Internet) 2006 (citado 2018 marzo 15) Recuperado de: http://cybertesis.unmsm.edu.pe/handle/cybertesis/1345

3. OMS Relación entre la satisfacción con el sistema de atención sanitaria y la experiencia personal de los pacientes.(Internet) 2017 (citado 2018 enero 23) Recuperado de: http://www.who.int/bulletin/volumes/87/4/07-050401-ab/es/

4. González, R.M., Fernández, P., González, L. La calidad de la atención en salud. (Internet) 2008 (citado 2017 junio 27) Recuperado de: http://www.medigraphic.com/pdfs/oral/ora-2008/oras081a.pdf

5. Santana, F., Bauer, A., Minamisava, R., Queiroz, A. \& Gomes, M. Calidad de los cuidados de enfermería y satisfacción del paciente atendido en un hospital de enseñanza. (Înternet) 2014 (citado 2017 jul 18) Recuperado de: http://www.scielo.br/pdf/rlae/v22n3/es_0104-1169rlae-22-03-00454.

6 Ortegón, C. Satisfacción del paciente post-quirúrgico en el área de hospitalización. (Tesis de Maestría en ciencias de enfermería). Universidad Autonoma de Nuevo Leon. 2005. 